\title{
Emergence of a prominent myeloid clone in a ZNF384-rearranged B-cell precursor acute lymphoblastic leukaemia post-corticosteroid pre-phase therapy.
}

\author{
Alpeshkumar Kapadia ${ }^{1}$, Sreejesh Sreedharanunni ${ }^{1}$, Safal Muhammed ${ }^{1}$, Indrani Karmakar ${ }^{1}$, \\ Sonia Rana ${ }^{1}$, Prashant Sharma ${ }^{1}$, Man Updesh Sachdeva ${ }^{1}$, and Amita Trehan ${ }^{1}$ \\ ${ }^{1}$ Post Graduate Institute of Medical Education and Research
}

May 12, 2020

\begin{abstract}
A 2-year-old girl with TCF3-ZNF384 re-arranged standard-risk B-cell precursor acute lymphoblastic leukaemia (BCP-ALL) showed an apparent lineage-switch to predominantly myeloid blasts following 7-days of corticosteroid therapy. ZNF384-rearranged leukemias are increasingly recognized to present either as mixed-phenotype acute leukemia or as BCP-ALL with pro-B immunophenotype. Lineage switch is a rare phenomenon described at relapse or following CAR-T-cell therapy previously mostly in KMT2A re-arranged leukemias; and now, also in ZNF384-rearranged patients. It is previously unreported in any patient after pre-phase corticosteroid therapy. Hematologists should be aware of this distinct emerging entity among leukemias with multi-lineage differentiation potential.
\end{abstract}

\section{Introduction}

ZNF384 (zinc finger protein 384) re-arrangements are recently recognized cytogenetic abnormalities that occur in as many as $48 \%$ of mixed-phenotype acute leukaemia (MPAL), B/myeloid and $4 \%$ of B-cell precursor acute lymphoblastic leukaemia (BCP-ALL) ${ }^{1}$. Several fusion partners of ZNF384 have been described, the most common being TCF3, but also EP300, ESWR1, TAF15, CREBBP, ARID1B, SYNRG and BMP2K ${ }^{1-3}$ We report a challenging case with TCF3-ZNF384 rearrangement that initially presented as BCP-ALL but showed the emergence of a large myeloid blast subset following one week of high-dose steroid therapy.

\section{Case History}

A two-and-a-half-year-old female child presented with fever and pallor of 1-week duration. Examination revealed hepatomegaly (span $11 \mathrm{~cm}$ ) and splenomegaly $(2 \mathrm{~cm}$ below the left subcostal margin). The hemoglobin was $46 \mathrm{~g} / \mathrm{L}$, platelet count $14.0 \times 10^{9} / \mathrm{L}$ and total leucocyte count $14.7 \times 10^{9} / \mathrm{L}$. Peripheral blood film showed $62 \%$ circulating blasts (Fig. 1A-B ). Bone marrow aspiration revealed $82 \%$ blasts with scanty, agranular basophilic cytoplasm without Auer rods; $2 \%$ of the blasts showed cytochemical myeloperoxidase positivity. On flow cytometry, the blasts expressed bright CD19 and dim CD10 along with cytoplasmic CD79a and CD22. There was cross-lineage antigen expression of CD33 (bright; $90 \%$ cells) and CD13 (dim; 14\% cells). The blasts were also positive for CD34, HLA-DR, CD38, CD81 and CD86; and were negative for CD20, CD117, T-lineage associated markers, monocytic \& megakaryocytic-lineage associated markers. Cytoplasmic myeloperoxidase (anti-MPO) expression was also not noted. A working diagnosis of BCP-ALL with aberrant CD33 expression was rendered, following which the child was initiated on the Standard Risk (SR) BCP-ALL protocol of the Indian Childhood Collaborative Leukemia Group (ICiCLe), comprising of a pre-phase of prednisolone $60 \mathrm{mg} / \mathrm{m}^{2} / \mathrm{d}$ in three divided doses. ${ }^{4}$ 
Fluorescent in-situ hybridization (FISH) testing with dual-colour dual-fusion probes for $B C R$ - $A B L 1$ and ETV6-RUNX1, dual break-apart probes for KMT2A and TCF3 (CytoTest Inc. Rockville, United States) revealed presence of TCF3 translocation in more than $80 \%$ of cells (Fig. 1E) . Subsequently, both TCF3PBX1 and TCF3-HLF translocations were excluded using a tri-colour probe (Cytocell Ltd, Cambridge).

After one week of pre-phase steroid therapy, although the toddler clinically appeared well, her leukocyte count increased to $63 \times 10^{9} / \mathrm{L}$ with $60 \%$ circulating blasts. On peripheral blood smear examination, in contrast to the morphology at diagnosis, these blasts were larger with indented nuclei, prominent nucleoli and moderate amount of granular basophilic cytoplasm without Auer rods. The blasts showed unequivocal cytochemical myeloperoxidase positivity in $20 \%$ of the cells (Fig. 1C-D ). The smaller lymphoblasts noted at the time of diagnosis were absent. Repeat flow cytometry performed on peripheral blood showed that $90 \%$ of the blasts expressed myeloid antigens (CD13, CD33, CD64, CD14, CD36, CD15 and cytoplasmic myeloperoxidase. The remaining $10 \%$ blasts showed the initial immunophenotype of BCP-ALL. The initial cytochemical myeloperoxidase and anti-MPO (flow cytometry) results were reviewed and the negative report $(<3 \%$ positivity) was reverified.

Repeat FISH testing revealed the TCF3 rearrangement in blasts as well as granulocytes (Fig. 1F) . In view of absence of metaphase, further FISH testing was performed using a commercially available dualcolour break-apart probe (ZytoVision GmbH, Germany) that confirmed rearrangement of ZNF384 (Fig. 1G). The diagnosis was revised to TCF3-ZNF384 [t(12;19)(p13;p13)] rearranged MPAL, B/myeloid. The patient's risk stratification was upgraded to High-Risk (HR) and appropriate management initiated ${ }^{4}$.

Her remaining induction phase chemotherapy was interrupted by two episodes of febrile neutropenia, both managed with antimicrobials. End-of-induction bone marrow revealed $03 \%$ blasts and no measurable residual disease (MRD) on high-resolution multiparameter flow cytometry. She had a stormy course prior to consolidation too, developing a fungal brain abscess requiring surgical drainage, leading to a 2-week delay in start of consolidation therapy. Currently, the patient is on an augmented BFM (Berlin-Frankfurt-Munster) consolidation protocol and continues her antifungal prophylaxis.

\section{Discussion}

TCF3-ZNF384 fusion gene resulting from a reciprocal translocation between chromosomes 19p13 (TCF3 ) and 12p13 (ZNF384) appears to be a distinct category. Bearing similarities toKMT2A-rearranged acute leukemias, these too present either as BCP-ALL or MPAL, B/myeloid type ${ }^{5}$ and are also associated with dim CD10 expression (pro-B immunophenotype) and upregulation of CD13 and/or CD33 $3^{1,2}$ as seen in our patient. About $2 \%$ blasts expressed myeloperoxidase, but the overall initial immunophenotype did not meet the required criteria for diagnosis of MPAL. Such diagnostic dilemmas with MPALs are frequent in routine practice and are also well-documented in the literature ${ }^{2}$. TCF3-ZNF384-positive BCP-ALLs are associated with higher total leukocyte counts, poor response to steroids at day- 8 and a relatively unfavourable outcome ${ }^{1}$. In our case too, the total leukocyte count showed a fivefold increase by day 8 , with a significant increase in myeloblasts.

A lineage switch, i.e. conversion from lymphoid to myeloid immunophenotype (and exceptionally, vice versa ) in acute leukemias is a rare phenomenon. It occurs more often in pediatric leukemias, being reported mostly in patients with KMT2Are-arrangement ${ }^{6-8}$ but occasionally also in patients with ZNF384 -rearranged acute leukemias ${ }^{5,9}$. The lineage switch mostly occurs at the time of relapse. However, it is also reported in patients who have received either CD19-targeting agents or chimeric antigen receptor T-cell therapy $7,8,10,11$.

Various mechanisms may explain the lineage switch from lymphoid to myeloid or emergence of large myeloid clone following one week of high-dose steroid therapy in our case. It could simply represent the unmasking of a myeloid sub-clone that was initially outnumbered by the preponderant lymphoid blasts. This is supported by the presence of $2 \%$ myeloperoxidase-positive blasts at diagnosis. An alternative explanation might lie in the inherent plasticity of leukaemia stem cells for divergent differentiation ${ }^{12}$. The presence ofZNF384 translocation initially noted in the lymphoid blasts and subsequently shown to be present also in granulocytic cells may points towards the origin of this leukemia from a primitive hematopoietic precursor with 
divergent differentiation potential. In support of this theory, gene expression profiles of ZNF384- rearranged BCP-ALLs as well as B/myeloid MPALs have revealed enrichment of stem cell pathways. ${ }^{2}$ The stem cell origin probably leads to an initial development of acute leukemia with single lineage differentiation; but a therapeutic challenge may unravel its multi-lineage differentiation potential.

In conclusion,ZNF384- rearranged leukemias are a unique and possibly under-recognized category of neoplasms with multi-lineage differentiation potential. Both hematopathologists as well as hemato-oncologists should be aware of the potential lineage switch that may occur in this group of leukemias.

Conflict of interest statement: - All authors declare that they do not have any conflict(s) of interest(s) to declare.

Acknowledgement: The authors are thankful to Dr Mayur Parihar, Tata Medical Centre for performing FISH testing for TCF3-HLF translocation.

\section{References:}

1. Hirabayashi S, Ohki K, Nakabayashi K, et al. ZNF384-related fusion genes define a subgroup of childhood B-cell precursor acute lymphoblastic leukemia with a characteristic immunotype.Haematologica . 2017;102:118-129.

2. Alexander TB, Gu Z, Iacobucci I, et al. The genetic basis and cell of origin of mixed phenotype acute leukaemia. Nature . 2018;562:373-379.

3. McClure BJ, Heatley SL, Kok CH, et al. Pre-B acute lymphoblastic leukaemia recurrent fusion, EP300ZNF384, is associated with a distinct gene expression. Br J Cancer . 2018;118:1000-1004.

4. Roy P, Narula G, Arora B, et al. Implementation of risk adapted therapeutic strategy for childhood acute lymphoblastic leukaemia - interim report of the pilot InPOG-ALL-15-01 study. Pediatr Hematol Oncol J . 2018;3:S19.

5. Oberley MJ, Gaynon PS, Bhojwani D, et al. Myeloid lineage switch following chimeric antigen receptor T-cell therapy in a patient with TCF3-ZNF384 fusion-positive B-lymphoblastic leukemia. Pediatr Blood Cancer . 2018;65:e27265.

6. Aldoss I, Song JY. Extramedullary relapse of KMT2A(MLL)-rearranged acute lymphoblastic leukemia with lineage switch following blinatumomab.Blood . 2018;131:2507.

7. Balducci E, Nivaggioni V, Boudjarane J, et al. Lineage switch from B acute lymphoblastic leukemia to acute monocytic leukemia with persistent $\mathrm{t}(4 ; 11)(\mathrm{q} 21 ; \mathrm{q} 23)$ and cytogenetic evolution under CD19-targeted therapy.Ann Hematol . 2017;96:1579-1581.

8. Rayes A, McMasters RL, O'Brien MM. Lineage Switch in MLL-Rearranged Infant Leukemia Following CD19-Directed Therapy. Pediatr Blood Cancer . 2016;63:1113-1115.

9. Grammatico S, Vitale A, La Starza R, et al. Lineage switch from pro-B acute lymphoid leukemia to acute myeloid leukemia in a case with $\mathrm{t}(12 ; 17)(\mathrm{p} 13 ; \mathrm{q} 11) / \mathrm{TAF} 15-\mathrm{ZNF} 384$ rearrangement. Leuk Lymphoma . 2013;54:1802-1805.

10. Zoghbi A, Zur Stadt U, Winkler B, Müller I, Escherich G. Lineage switch under blinatumomab treatment of relapsed common acute lymphoblastic leukemia without MLL rearrangement. Pediatr Blood Cancer . $2017 ; 64$.

11. Jacoby E, Nguyen SM, Fountaine TJ, et al. CD19 CAR immune pressure induces B-precursor acute lymphoblastic leukaemia lineage switch exposing inherent leukaemic plasticity. Nat Commun . 2016;7:12320.

12. Dorantes-Acosta E, Pelayo R. Lineage switching in acute leukemias: a consequence of stem cell plasticity? Bone Marrow Res . 2012;2012:406796.

Figure legends: 
Figure 1: (A-B) Morphology of blasts (May-Grünwald Giemsa stain; 1000x) and myeloperoxidase-staining (Hematoxylin counterstain) of blasts at diagnosis and; (C-D) at day-8 post-corticosteroid therapy. (E) Fluorescent in-situ hybridization using dual colour break apart probe showing deletion of telomeric end (3'end; orange) of TCF3 gene on chromosome 19p13.3 indicating unbalanced translocation in blasts and (F) granulocytes. (G)Fluorescent in-situ hybridization using dual colour break apart probe showing an abnormal signal pattern indicative of ZNF384translocation (DAPI counterstain).

Figure 2: Immunophenotype of blasts - (A-D) at diagnosis $\left(\mathrm{CD} 19^{\mathrm{pos}} \mathrm{CD} 10^{\mathrm{dimpos}} \mathrm{CD} 34^{\text {pos }} \mathrm{CD} 33^{\mathrm{pos}} \mathrm{CD} 13^{\mathrm{dim}}\right.$ $\left.\mathrm{CD} 15^{\text {neg }} \mathrm{CyCD} 79 \mathrm{a}^{\text {pos }} \quad \mathrm{CyCD} 22^{\text {pos }}\right) \quad(\mathbf{E}-\mathbf{H})$ post-corticosteroid prephase therapy $\left(\mathrm{CD} 19^{\text {neg }} \mathrm{CD} 10^{\text {neg }}\right.$ CD34 $\left.{ }^{\text {neg }} \mathrm{CD} 33^{\text {pos }} \mathrm{CD} 13^{\text {pos }} \mathrm{CD} 15^{\text {pos }} \mathrm{CyCD} 79 a^{\text {neg }} \mathrm{CyCD} 22^{\text {neg }}\right)$
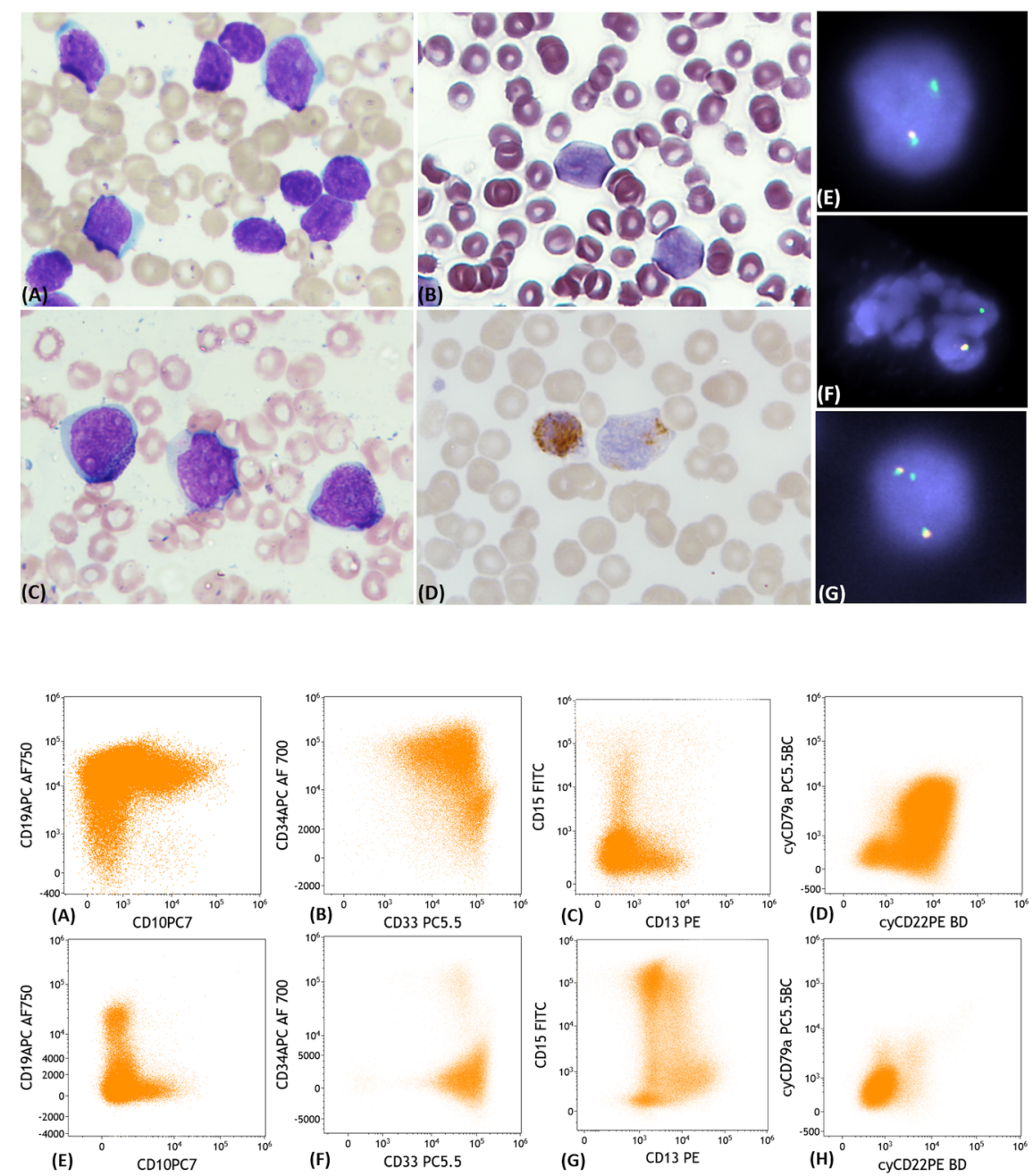